\title{
CAA Study of Airfoil Broadband Interaction Noise Using Stochastic Turbulent Vorticity Sources
}

\author{
Thomas Hainaut*, Gwénaël Gabard ${ }^{\dagger}$ and Vincent Clair \\ Institute of Sound and Vibration Research, University of Southampton, SO17 1BJ Southampton, UK.
}

The interaction of the turbulent wakes of the rotor with the outer guide vanes is one of the main broadband noise source in turbofan engines at approach conditions. Hence its prediction and reduction is a priority for engine manufacturers. The development of numerical methods is required as analytical approaches are limited to simple geometries and simplified flow configurations. The linearized Euler equations are solved in the time-domain to model the response of an isolated airfoil interacting with turbulence that is stochastically synthesized and injected in the computational domain through vorticity sources. This new method of injection has the advantages of being easy to implement and parallelize in an existing solver, whilst the generated turbulence is frozen. The method is firstly validated on a $2 \mathrm{D}$ free-field configuration. It is then applied, in the framework of the Fan Stage Broadband Noise Benchmarking Programme, to a two-dimensional NACA 65(12)10 airfoil with no angle of attack and the results are validated through comparisons with experimental data. Afterwards, the effect of the angle of attack is studied and the results suggest that a one-component turbulent model is not satisfactory to perform accurate acoustic predictions with an angle of attack, as it overestimates the rate of decay of the acoustic spectra at high frequencies. The study of the influence of the integral length scale of the turbulence confirms that the airfoil leading edge response is only modulated by the incoming turbulence characteristics. Finally, the acoustic spectra predicted for different velocities show a better agreement with a flat plate analytical model when the velocity is increased.

\section{Introduction}

In turbofan engines, the fan noise which is the combination of tonal and broadband components, is dominant at reduced thrust levels. ${ }^{1}$ It can be decomposed into the self-noise of the rotor and the guide vanes, the rotor-boundary layer interaction noise and the rotor wake guide vanes interaction noise. However, the fan noise is complex to reduce since the fan stage provides the majority of the total thrust. Therefore, noise reductions have to be achieved without degrading the performances.

The numerical study of broadband noise generated by a turbulent flow has always been demanding in computational resources due to the presence of a large variety of scales, from the small turbulent scales to the large acoustic length scales. Computational AeroAcoustics (CAA) methods are then often based on the use of high-order methods to reduce the number of grid points while ensuring the quality of the acoustic propagation. In the context of predicting broadband noise generated between the interaction of the turbulent wakes of the fan blades and the outlet guide vanes, one approach would be to consider the complete rotorstator stage or an angular sector including one or more blade/vane channels. However, correctly modeling the turbulent wakes of the rotor would require to solve the full governing equations, coupled with a fine mesh, and the computational cost associated makes it difficult to use in an industrial context.

An alternative methodology consists in restricting the configuration to the stator only, and introducing a synthetic turbulence in the computational domain. This allows a drastic reduction in the size of the computational domain and, as the problem is limited to the study of the interaction of the injected turbulence

${ }^{*} \mathrm{PhD}$ student, t.hainaut@soton.ac.uk

${ }^{\dagger}$ Associate professor, Senior member AIAA, gabard@soton.ac.uk

${ }^{\ddagger}$ Research fellow, v.j.clair@soton.ac.uk 
with the stator, the generation and propagation of the noise can be obtained without solving the full governing equations. Nonetheless, the incoming turbulence has to be modeled and injected in the computational domain. This could be achieved by the use of stochastic methods, as introduced in 1970 by Kraichnan ${ }^{2}$ where the fluctuating turbulent field is decomposed as a sum of Fourier modes. Nevertheless, this summation can become computationally expensive as the number of modes increases. A second solution is to filter white noise to reproduce imposed spatial and temporal correlations of the incoming turbulence, as proposed by Ewert et al. ${ }^{3}$ in the Random Particle Method (RPM) or by Dieste \& Gabard. ${ }^{4,5}$ Another alternative introduced by Jarrinet al., ${ }^{6,7,8}$ the Synthetic Eddy Method (SEM), is based on the decomposition of the turbulence in a sum of eddies, calibrated to match the desired features of the turbulence.

Leading edge noise has been studied by Dieste \& Gabard ${ }^{4,5,9}$ for a 2D flat plate at Mach 0.362 using a method similar to the RPM. They showed that the inclusion of the temporal evolution of turbulence in the model has a very limited effect on the acoustic radiation of the flat plate. This might be explained by the fact that the turbulence has a relatively long correlation time long compared to the time needed to convect along the flat plate. This observation indicates that generating a frozen turbulence should be satisfying to study interaction noise on a thin airfoil. Clair et al. ${ }^{10}$ developed a specific stochastic model to generate a frozen turbulent velocity field from a sum of Fourier modes, taking inspiration from Amiet's model ${ }^{11}$ to reduce the dimension of the model, and applied it to 3D airfoils. A similar method has also been developed by Gill et al. ${ }^{12}$ to assess the effect of the airfoil thickness.

The present paper proposes a new method to inject turbulence in the computational domain, by using localized vorticity sources. It allows the turbulence to be injected anywhere in the domain. However, since the vorticity sources are localized, the turbulent fluctuations are only convected by the mean flow, leading to a frozen turbulent field. The method is first validated in two-dimensions on a NACA 65(12)-10 airfoil with no angle of attack, which is part of the Fan Stage Broadband Noise Benchmarking Programme. ${ }^{13}$ Then, the effect of the angle of attack, mean flow velocity, and integral length scale of the turbulence on the radiated noise are studied.

\section{Synthetic turbulence injected through vorticity sources}

The propagation of small amplitude perturbations on a mean flow can be described by the linearized Euler equations (LEE), written here in a non-conservative form:

$$
\left\{\begin{array}{l}
\frac{\partial \rho^{\prime}}{\partial t}+\mathbf{u}_{0} \cdot \nabla \rho^{\prime}+\mathbf{u}^{\prime} \cdot \nabla \rho_{0}+\rho_{0} \nabla \cdot \mathbf{u}^{\prime}+\rho^{\prime} \nabla \cdot \mathbf{u}_{0}=0 \\
\frac{\partial \mathbf{u}^{\prime}}{\partial t}+\left(\mathbf{u}_{0} \cdot \nabla\right) \mathbf{u}^{\prime}+\left(\mathbf{u}^{\prime} \cdot \nabla\right) \mathbf{u}_{0}+\frac{\nabla p^{\prime}}{\rho_{0}}-\frac{\nabla p_{0} \rho^{\prime}}{\rho_{0}^{2}}=\mathbf{S}_{v} \\
\frac{\partial p^{\prime}}{\partial t}+\mathbf{u}_{0} \cdot \nabla p^{\prime}+\mathbf{u}^{\prime} \cdot \nabla p^{\prime}+\gamma p_{0} \nabla \cdot \mathbf{u}^{\prime}+\gamma p^{\prime} \nabla \cdot \mathbf{u}_{0}=0
\end{array}\right.
$$

where $\rho, p, \mathbf{u}$ and $\gamma$ denote the density, pressure, velocity and specific heat ratio, respectively. The mean flow variables are denoted by the subscript ${ }_{0}$ and the perturbation variables by the superscript ${ }^{\prime}$. The right-hand side of the momentum equation $\mathbf{S}_{v}$ denotes a vorticity source. The LEE can be used as governing equations to study leading edge noise generation and acoustic wave propagation since they are able to support vortical, entropy and acoustic modes. ${ }^{14}$

In the present paper, the CAA solver PIANO (Perturbation Investigation of Aerodynamic NOise) developed by the DLR (German Aerospace Center) is used. It solves the LEE in the time domain using a $4^{\text {th }}$-order DRP (Dispersion Relation Preserving) finite difference scheme with a 7-point stencil proposed by Tam \& Webb, ${ }^{15}$ which has been designed to minimize the numerical dispersion introduced by the discretization. The time integration is performed using a $4^{\text {th }}$-order Runge-Kutta scheme with 4 -stage. A $8^{\text {th }}$-order selective filter is also applied in order to prevent high-frequency spurious oscillations to appear in the computational domain.

\section{II.A. Injection of turbulent fluctuations through local sources}

In this work, the synthetic turbulence is injected in the domain through localized vorticity sources. As mentioned before, the turbulent perturbations are then convected by the mean flow, leading to a frozen turbulent field. 
We assume a vorticity source injected in a uniform mean flow, away from any other source, hence: $\rho^{\prime}=0$, $p^{\prime}=0$ and $\nabla \cdot \mathbf{u}^{\prime}=0$. Following those assumptions, one can simplify the LEE to obtain a transport equation:

$$
\left(\frac{\partial}{\partial t}+u_{0} \frac{\partial}{\partial x}\right) \mathbf{u}^{\prime}=\mathbf{S}_{v}(\mathbf{x}, t)
$$

where the vorticity source term $\mathbf{S}_{v}(\mathbf{x}, t)$ can be decomposed in a spatial distribution $\mathbf{g}(\mathbf{x})$ and a temporal signal $s(t)$ so that $\mathbf{S}_{v}(\mathbf{x}, t)=\mathbf{g}(\mathbf{x}) s(t)$.

The spatial Fourier transform of the spatial component will affect the frequency spectrum of the velocity fluctuations convected downstream of the source. Thus the frequency spectrum of the time component $s(t)$ injected through the local source has to be modified to ensure that the desired spectrum is obtained for the turbulence convected downstream of the source. The frozen turbulence hypothesis implies $k_{x}=\omega / u_{0}$, thus the spectral density $S_{22}(\omega)$ of the time signal $s(t)$ writes:

$$
S_{22}(\omega)=\frac{\left|u_{0}\right|}{\pi^{2}} \frac{\Phi_{22}\left(\omega / u_{0}\right)}{\left|\hat{g}_{2}\left(\omega / u_{0}, y_{c}\right)\right|^{2}},
$$

where $\Phi_{22}\left(\omega / u_{0}\right)$ is the desired wavenumber spectrum of the fluctuating velocity component normal to the chord and $\hat{g}_{2}\left(k_{x}=\omega / u_{0}, y_{c}\right)$ is the Fourier transform of the spatial component of the source $\mathbf{g}(\mathbf{x})$, on the streamwise direction at the position $y=y_{c}$ defined in the following section.

For a prescribed wavenumber spectrum $\Phi_{22}\left(\omega / u_{0}\right)$, the spectral density $S_{22}(\omega)$ is calculated and then used to generate a time signal $s(t)$. Several methods had been proposed in the literature for this purpose, for instance for generating non-Gaussian signals, ${ }^{16}$ or using wavelets. ${ }^{17}$ The method used here is based on a Fourier transform. ${ }^{18}$ Finally, the resulting temporal signal $s(t)$ is used to form the vorticity source $\mathbf{S}_{v}(\mathbf{x}, t)=\mathbf{g}(\mathbf{x}) s(t)$.

\section{II.B. Gaussian vorticity source}

The spatial distribution of the vorticity source used in this paper is defined as the curl of a Gaussian vector potential $\psi(\mathbf{x})$ which ensures divergence-free velocity perturbations and is defined as:

$$
\mathbf{g}(\mathbf{x})=\nabla \times \boldsymbol{\psi}(\mathbf{x})=\nabla \times\left(A b \sqrt{\frac{e}{\ln (4)}} \exp \left[-\ln (2) \frac{\left|\mathbf{x}-\mathbf{x}_{c}\right|^{2}}{b^{2}}\right] \mathbf{e}_{z}\right),
$$

where $b$ is the half-value radius of the Gaussian, $A$ the amplitude, $\mathbf{x}_{c}=\left(x_{c}, y_{c}\right)$ the coordinates of the center of the Gaussian and $\mathbf{e}_{z}$ the axis of rotation.

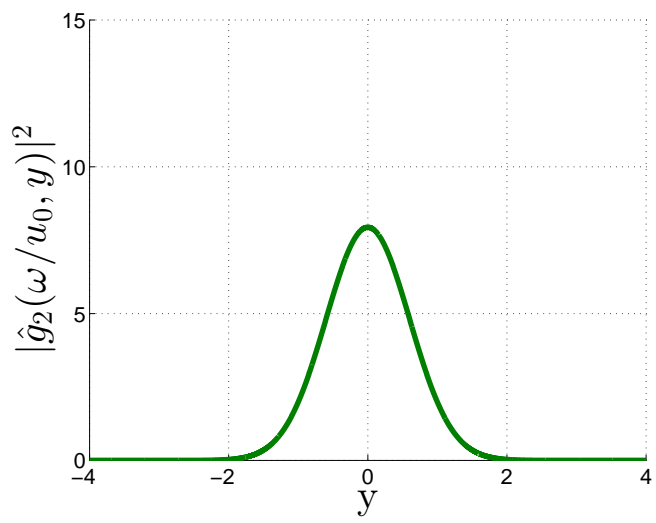

(a) Single vorticity source, $\mathbf{x}_{c}=(0,0)$.

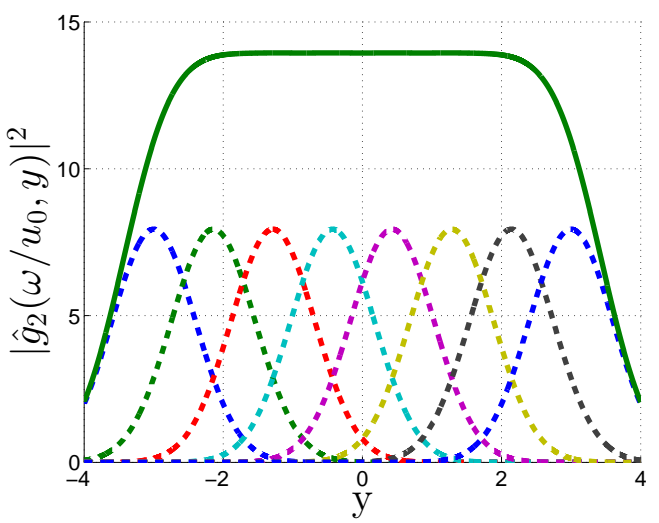

(b) Eight incoherent vorticity sources, evenly spaced in the $\mathrm{y}$-direction between $-3 \leq y \leq 3$.

Figure 1: Amplitude of $\left|\hat{g}_{2}\left(\omega / u_{0}, y\right)\right|^{2}$ at an angular frequency $\omega_{0}$ as a function of the y-direction (-). The dashed lines in Figure (b) represents the contribution of each incoherent vorticity source.

The evolution of the streamwise Fourier transform of the spatial component of the vorticity source $\left|\hat{g}_{2}\left(\omega / u_{0}, y\right)\right|$ in the normal-direction, at a given angular frequency $\omega_{0}$, plotted in Figure 1a, shows a Gaussian 
shape with a maximum at $y=y_{c}$. This means that the velocity field injected in the domain using the method presented in the previous section will fade away from $y=y_{c}$. It is possible to construct a spatial distribution of the source to keep a constant value of $\left|\hat{g}_{2}\left(\omega / u_{0}, y\right)\right|$, and thus of the velocity magnitude, over a chosen spatial extent in the normal-direction by superposing $N_{s}$ evenly spaced Gaussian distributions, as plotted in Figure 1b. By doing so, the resulting energy is increased when setting multiple vorticity sources compared to a single source. Assuming coherent sources, to retain the same amount of energy, the amplitude parameter $A$ can be corrected:

$$
A=\left[\sum_{i}^{N_{s}} \exp \left(-\frac{\ln 2}{b^{2}}\left(y_{c}^{(0)}-y_{c}^{(i)}\right)^{2}\right)\right]^{-1},
$$

where $y_{c}^{(0)}$ and $y_{c}^{(i)}$ are the vertical component of the center of respectively the single source and the $i^{\text {th }}$ source. On the other hand, if each source uses a different realization of the temporal signal $s(t)$ (incoherent sources), then $A$ becomes:

$$
A=\left[\sum_{i}^{N_{s}}\left(\exp \left(-\frac{\ln 2}{b^{2}}\left(y_{c}^{(0)}-y_{c}^{(i)}\right)^{2}\right)\right)^{2}\right]^{-1} .
$$

\section{II.C. Validation of the method}

To validate the generation of broadband gusts using a vorticity source, a free-field simulation with a uniform mean flow is realized. To get the desired wavenumber spectrum $\Phi_{22}\left(\omega / u_{0}\right)$ in the domain, a time signal $s(t)$ with a spectral density $S_{22}(\omega)$ given by equation (3) is generated in order to form the source term $S_{v}$. The computational domain extends between $0 \leq x \leq 10$ and $-2 \leq y \leq 2$. The domain is discretized with a uniform spacing $\Delta x=\Delta y=0.02$. The vorticity source is centered at $\mathbf{x}_{c}=(2,0)$ and a velocity sensor is located at $\mathbf{x}_{M}=(7.5,0)$ to record the velocity fluctuations as the gusts convect. The non-dimensionalized variables of the problem are the static density $\rho_{\infty}^{*}=1$, the static speed of sound $a_{\infty}^{*}=1$ and a length $L^{*}=1$. The mean flow velocity is uniform, oriented in the x-direction, with a Mach number $M=0.5$.

The Gaussian distribution defining the vorticity source has a half-radius $b=0.16$ and an amplitude $A=1$. Tam outflow boundary condition ${ }^{15}$ is used on the downstream boundary, and Tam radiation boundary condition $^{15}$ on the others. The non-dimensionalized time step is set to $t^{*}=0.012$, which gives a CFL number of 0.9 , and the simulation is run over $2^{19}$ iterations. Such a large number of iterations is used for validation purposes, to average the wavenumber spectrum on multiple segments by performing a periodogram. To prevent spurious oscillations to develop in the domain, a $8^{\text {th }}$-order explicit filter is applied at every time step.

For validation purposes, the chosen wavenumber spectrum is a white noise defined by:

$$
\Phi_{22}\left(\omega / u_{0}\right)=\frac{1}{2}\left[\mathcal{H}\left(\omega / u_{0}-\omega_{\min } / u_{0}\right)-\mathcal{H}\left(\omega / u_{0}-\omega_{\max } / u_{0}\right)\right]
$$

where $\mathcal{H}$ is the Heaviside function and $\left(\omega_{\min } / u_{0}, \omega_{\max } / u_{0}\right)$ are the lower and upper limits of the white noise, with respectively $\omega_{\min }=\pi / 4 \mathrm{rad} . \mathrm{s}^{-1}$ and $\omega_{\max }=2 \pi \mathrm{rad} . \mathrm{s}^{-1}$. The prescribed wavenumber spectrum is plotted in Figure 2a and the resulting spectral density is plotted in Figure 2b. One realization of the random signal $s(t)$, realized using a method based on the Fourier transform, ${ }^{18}$ is plotted in Figure 2c.

The computation times is about 4 hours on 8 Intel ${ }^{\circledR}$ i7-3770 processor cores with a core frequency of 3.4GHz. The instantaneous y-velocity fluctuation is plotted in Figure 3a. It shows the turbulence generated at the vorticity source location and convected downstream by the uniform mean flow. The solution does not seem to be contaminated by reflections on the downstream boundary. Moreover, the turbulence is frozen, hence using the relation $S_{\text {sensor }}\left(\omega=k u_{0}\right)=\Phi\left(\omega / u_{0}\right) / u_{0}$, the measurement of the instantaneous velocity perturbations allow the computation of the turbulence wavenumber spectrum. The spectral density of the turbulent velocity is calculated using a Welch method with 80 segments and an overlapping of $10 \%$. It allows a reduced noise in the estimated power spectrum in exchange for reducing the frequency resolution, compared to standard periodogram methods. The derived wavenumber spectrum is plotted in Figure $3 \mathrm{~b}$. A good agreement is shown between the desired and simulated spectrum. The lower and upper frequency limits of the white noise are respected, as well as the amplitude. 


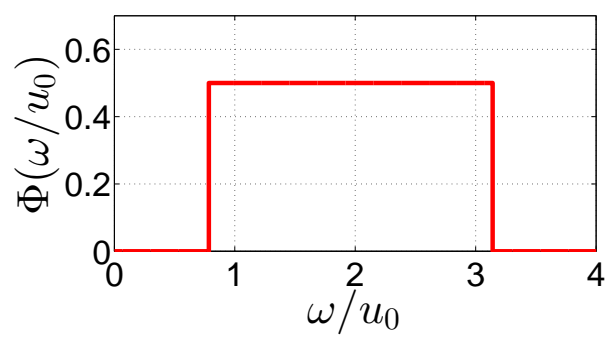

(a) Desired two-sided wavenumber spectrum of the fluctuating velocity $\Phi_{22}\left(\omega / u_{0}\right)$

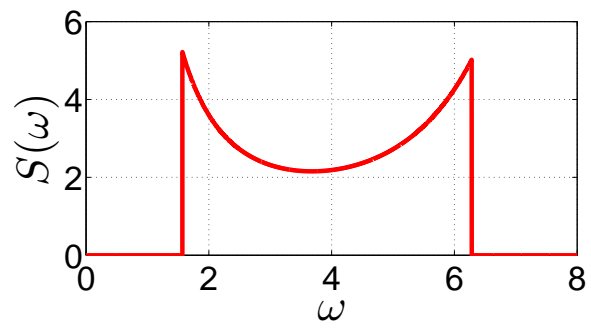

(b) Corresponding two-sided spectral density of the temporal signal $S_{22}(\omega)$

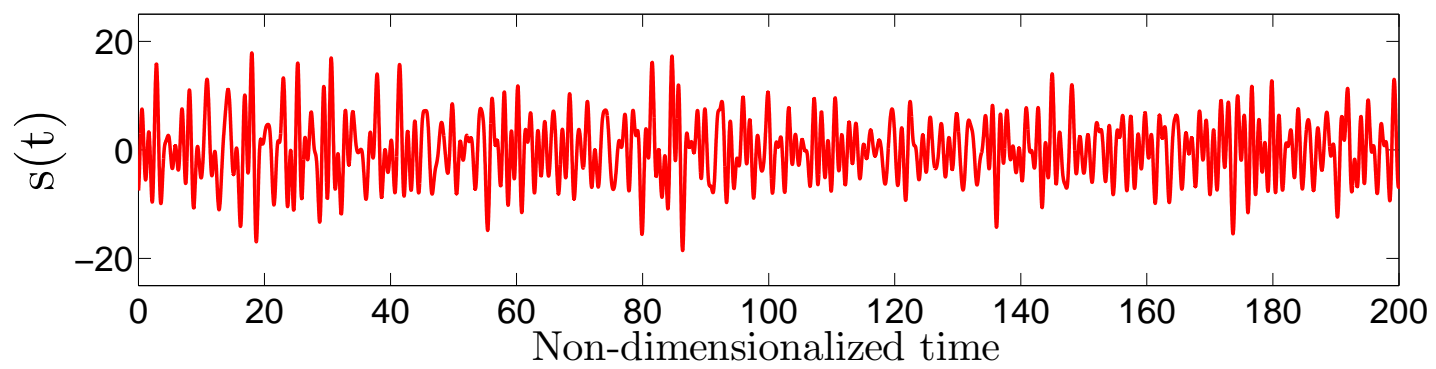

(c) One realization of the random signal $s(t)$

Figure 2: Generation of the temporal signal $s(t)$ for the vorticity source.

\section{Validation}

The validation case is the Fundamental test Case 1 (FC1) in the Fan Stage Broadband Noise Benchmarking Programme. ${ }^{13}$ It focuses on the noise generated by a turbulent velocity field impinging an isolated NACA 65(12)-10 airfoil in two-dimensions. The chord of the airfoil is $c=0.15 \mathrm{~m}$, at an angle of attack $\alpha=15^{\circ}$, and the incoming free stream velocity is $u_{\infty}=60 \mathrm{~m} \cdot \mathrm{s}^{-1}$.

\section{III.A. Mean flow}

As the mean flow defines the streamlines along which the velocity fluctuations are convected, a correct estimation is required. All the mean flow simulations presented in this paper have been done performed within the commercial software ANSYS Fluent ${ }^{\mathrm{a}}$, using the pressure-based solver, with a second-order spatial discretization.

In a CAA simulation, the presence of shear layers, boundary layers or significant velocity gradients can be a source of linear instabilities in the LEE. In the present paper, three configurations have been studied for the estimation of the mean flow.

The first configuration includes the presence of the jet of the wind tunnel, as it has been shown to have an influence on the loading of the airfoil. ${ }^{19,20}$ This effect of the jet is assessed in the present study by performing a second calculation for the airfoil in free stream. In this case, there is no deflection of the flow as in the jet configuration, so the angle of attack is corrected to $\alpha=4.21^{\circ}$ accordingly to ref. [21]. These two calculations are performed by solving the RANS equations, thus, the boundary layer developing on the surface of the airfoil will induce strong velocity gradients. When interpolated on the CAA mesh (see section III.B.1) where the cell size in the vicinity of the airfoil is large compared to the RANS mesh, these gradients can introduce instabilities leading to the divergence of the computation. To tackle this issue, the third configuration considered is similar to the second one (free stream), but Euler's equations are solved instead of RANS. Since the flow is allowed to slip on the airfoil surface with Euler's equations, the velocity gradients in this region are expected to be less important. The meshes are designed to ensure a cell size

\footnotetext{
${ }^{a}$ ANSYS Fluent : www.ansys.com
} 


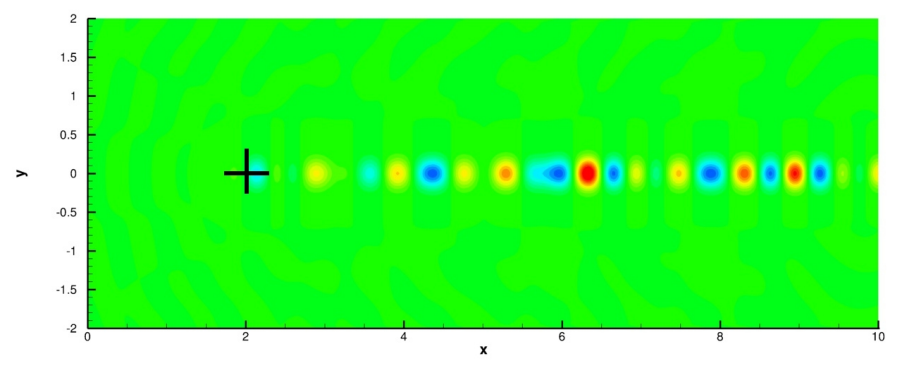

(a) Instantaneous transverse-velocity perturbations. The black cross denotes the localization of the vorticity source. Levels in between $\pm 5 \mathrm{~m} / \mathrm{s}$.

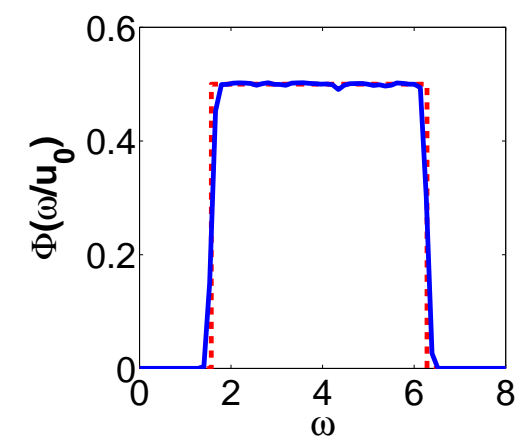

(b) Wavenumber spectrum $\Phi_{22}\left(\omega / u_{0}\right)$ prescribed $(-\mathbf{-})$ and measured $(\square)$.

Figure 3: Instantaneous velocity perturbations and wavenumber spectrum $\Phi_{22}\left(\omega / u_{0}\right)$ inside the computational domain.

at the wall such that the dimensionless wall distance ${ }^{\mathrm{b}} y^{+} \leq 1$. The cells are stretched with an expansion ratio less than $15 \%$ and the grids extend up to 30 chords in the normal and downstream directions. For the free stream cases, the upstream extent is also 30 chords, whereas it is only one chord for the configuration including the jet due to the presence of the nozzle (note also that the nozzle has a $0.15 \mathrm{~m}$ height). The meshes contain about 130000 points for the jet configuration and 75000 points for the free stream configurations. The parameters of the different meshes are summarized in Table 1 . The turbulence model used for the calculations is a SST $k-\omega$ model. The static density is set to $\rho_{\infty}=1.2 \mathrm{~kg} / \mathrm{m}^{3}$, the static speed of sound to $c_{\infty}=340 \mathrm{~m} / \mathrm{s}$ and the static turbulent viscosity ratio to approximately 10 .

\begin{tabular}{|l||c|c|c|c|c|}
\hline Configuration & Eq. solved & $\alpha$ & x-extent & y-extent & \# of points \\
\hline Jet & RANS & $15^{\circ}$ & $-c \leq x \leq 30 c$ & $-30 c \leq x \leq 30 c$ & 131576 \\
free stream 1 & RANS & $4.21^{\circ}$ & $-30 c \leq x \leq 30 c$ & $-30 c \leq x \leq 30 c$ & 76252 \\
Free stream 2 & Euler & $4.21^{\circ}$ & $-30 c \leq x \leq 30 c$ & $-30 c \leq x \leq 30 c$ & 76252 \\
\hline
\end{tabular}

Table 1: Parameters of the different meshes for the 3 configurations considered for the mean flow calculations.

The evolution of the normal cell size in wall units $\left(y^{+}\right)$along the airfoil surface, plotted in Figure 4 for the two configurations where the RANS equations are solved, is shown to have values inferior or close to unity.

The pressure coefficient obtained in the jet configuration, have a good agreement compared (on Figure 5) with the experiments conducted by Gruber et al., ${ }^{22,21}$ where the nozzle outflow is of height $0.15 \mathrm{~m}$ and at a distance of $0.15 \mathrm{~m}$ of the leading edge.

The mean velocity in the x-direction and pressure fields are shown in Figure 6, 7 and 8, respectively for the RANS with the jet, the RANS in free-field and the Euler in free-field simulations. The confinement effect due to the presence of the jet is clearly visible in Figure 6 compared to the free stream configurations. In the Euler computation, the absence of boundary layer is observable, especially on the extrados, as well as the absence of wake downstream of the airfoil.

To perform CAA simulations using a viscous mean flow despite having a boundary layer on the profile, the value of the first four cells of the CAA mesh, starting from the profile, are duplicated in order to reduce the velocity gradient, and thus potential linear instabilities. This technique have already being used ${ }^{10,23}$ with no noticeable effect on the radiated acoustic.

One should also note that, in this paper, the turbulence generated has only one-component, yet Gill ${ }^{12}$ showed it is not satisfactory as it overestimates the rate of decay at high frequency, which is confirmed in the section IV.A. As a result, a profile with no incidence is studied.

\footnotetext{
${ }^{\mathrm{b}} y^{+}$defined as $y^{+} \equiv\left(y u_{*}\right) / \nu$ with $y$ the distance to the nearest wall, $u_{*}$ the friction velocity at the nearest wall and $\nu$ the local kinetic viscosity.
} 


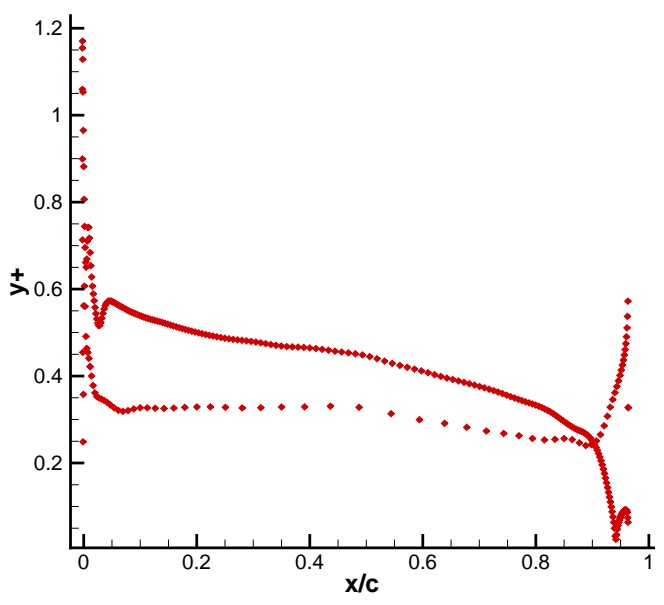

(a) Jet configuration

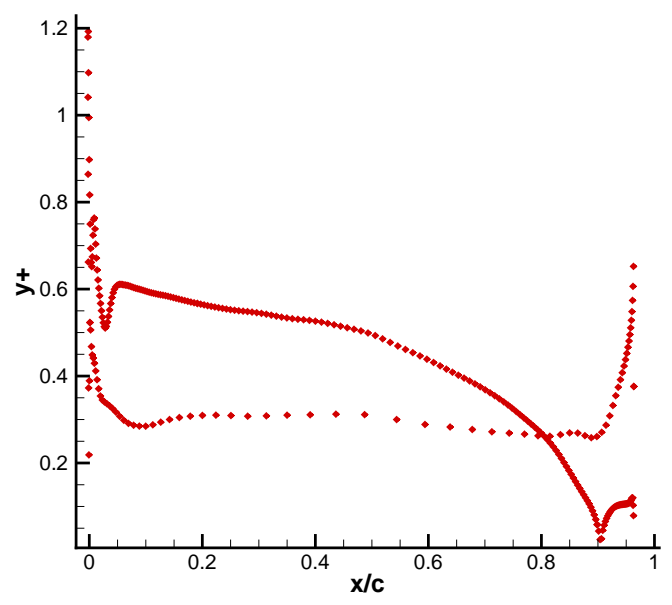

(b) Freestream configuration

Figure 4: Evolution of the normal cell size at the airfoil surface in wall units $\left(y^{+}\right)$(a) in the jet configuration and (b) in free stream configurations.

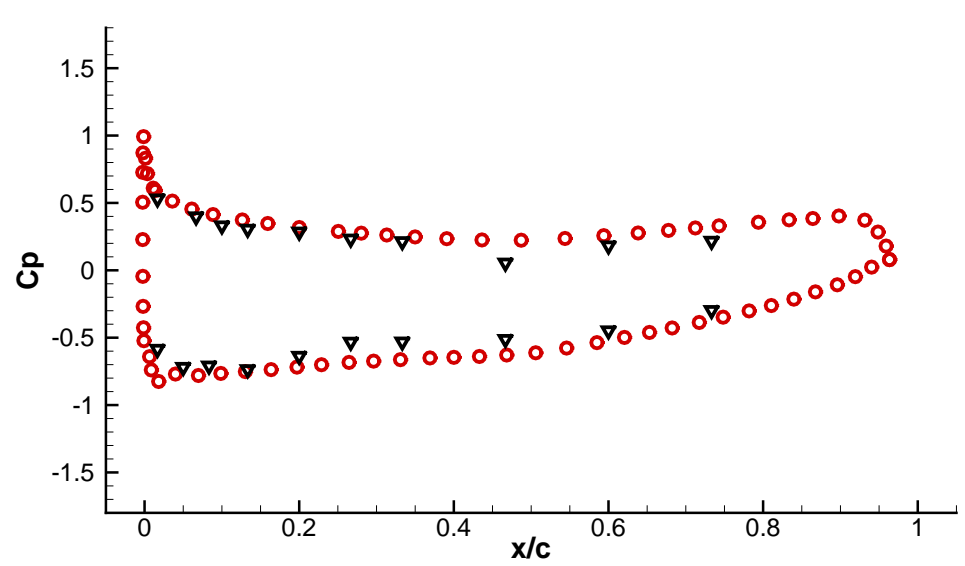

Figure 5: Comparison of the pressure coefficient along the airfoil between the RANS simulation in a jet configuration (०) and the experiments conducted by Gruber et al. ${ }^{22,21}(\nabla)$.

\section{III.B. Aeroacoustic simulation}

\section{III.B.1. Mesh}

The mesh has been designed to support acoustic waves up to a maximum frequency $f_{\max }=10000 \mathrm{~Hz}$, corresponding to a Strouhal number ${ }^{\mathrm{c}} S t_{\max }=25$, over a distance of two chords away from the airfoil in every direction. The velocity perturbations introduced in the domain are also supported by the mesh, but only from the injection plane to the trailing edge of the airfoil. The total extent of the mesh is however larger as it goes up to 20 chords away from the profile, which slowly dissipate the higher frequencies before reaching the boundaries. For the larger wavelengths, Tam's radiation boundary condition ${ }^{15}$ is applied on the upstream, top and bottom boundaries of the computational domain, and Tam's outflow boundary condition ${ }^{15}$ on the outflow boundary. These boundaries conditions are derived from asymptotic solutions of the linearized Euler equations to let acoustic waves leave the domain while minimizing reflections. The outflow boundary

\footnotetext{
${ }^{\mathrm{c}}$ Strouhal number defined as St $=(f c) / u$ with $f$ the frequency, $c$ the chord of the airfoil and $u$ the free stream velocity
} 

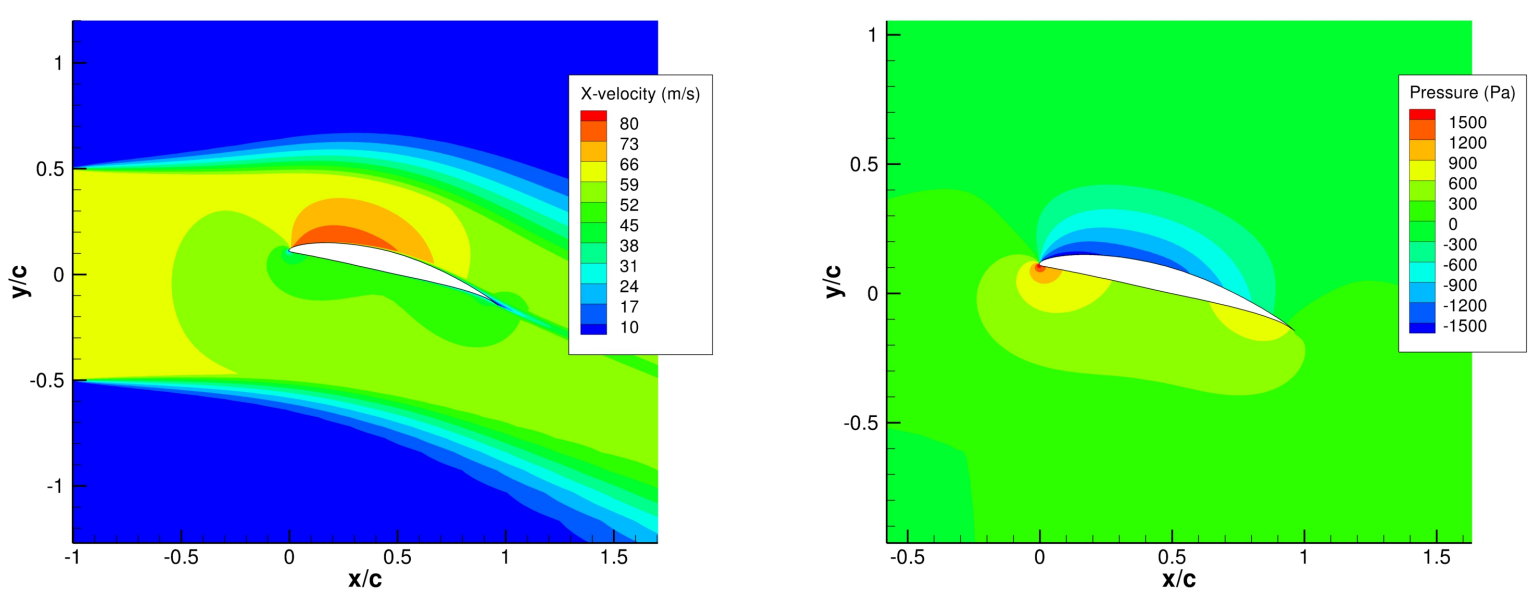

Figure 6: RANS solution on the jet configuration. (a) Velocity in the $x$-direction and $(b)$ pressure variations around $p_{\infty}=101325 \mathrm{~Pa}$.
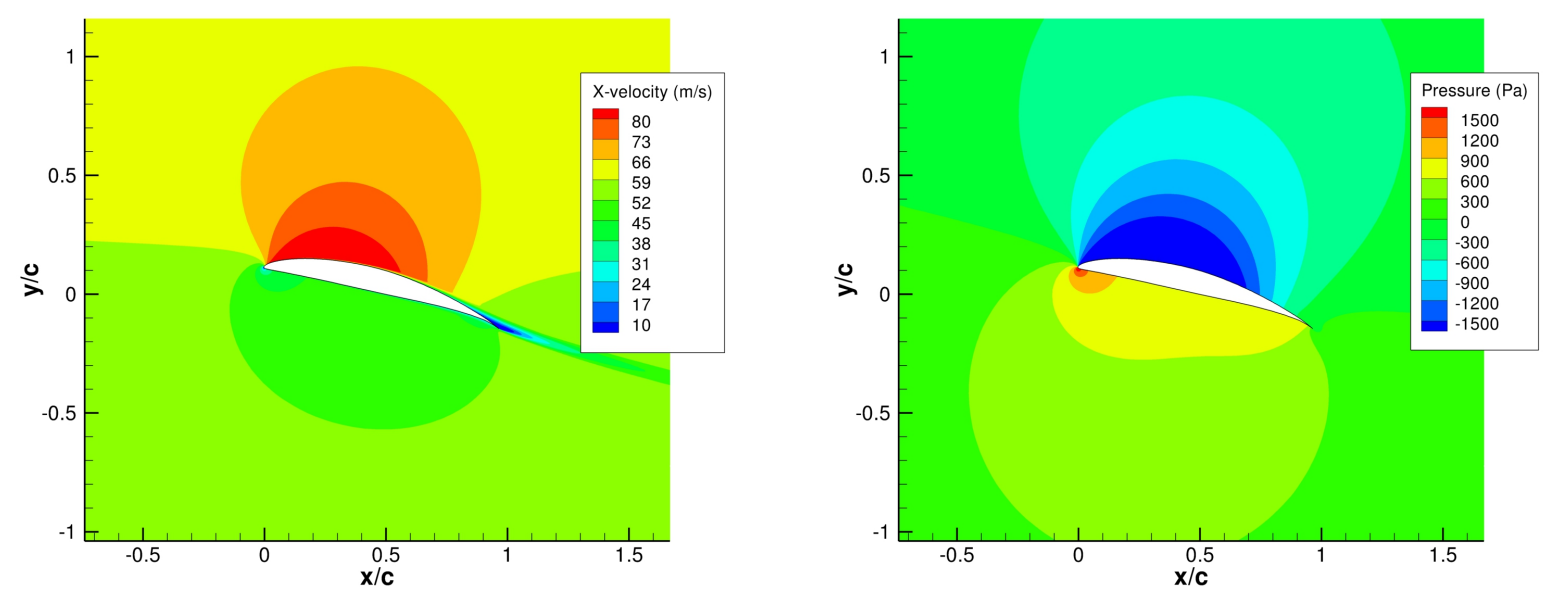

Figure 7: RANS solution on the free stream configuration. (a) Velocity in the $x$-direction and $(b)$ pressure variations around $p_{\infty}=101325 \mathrm{~Pa}$.
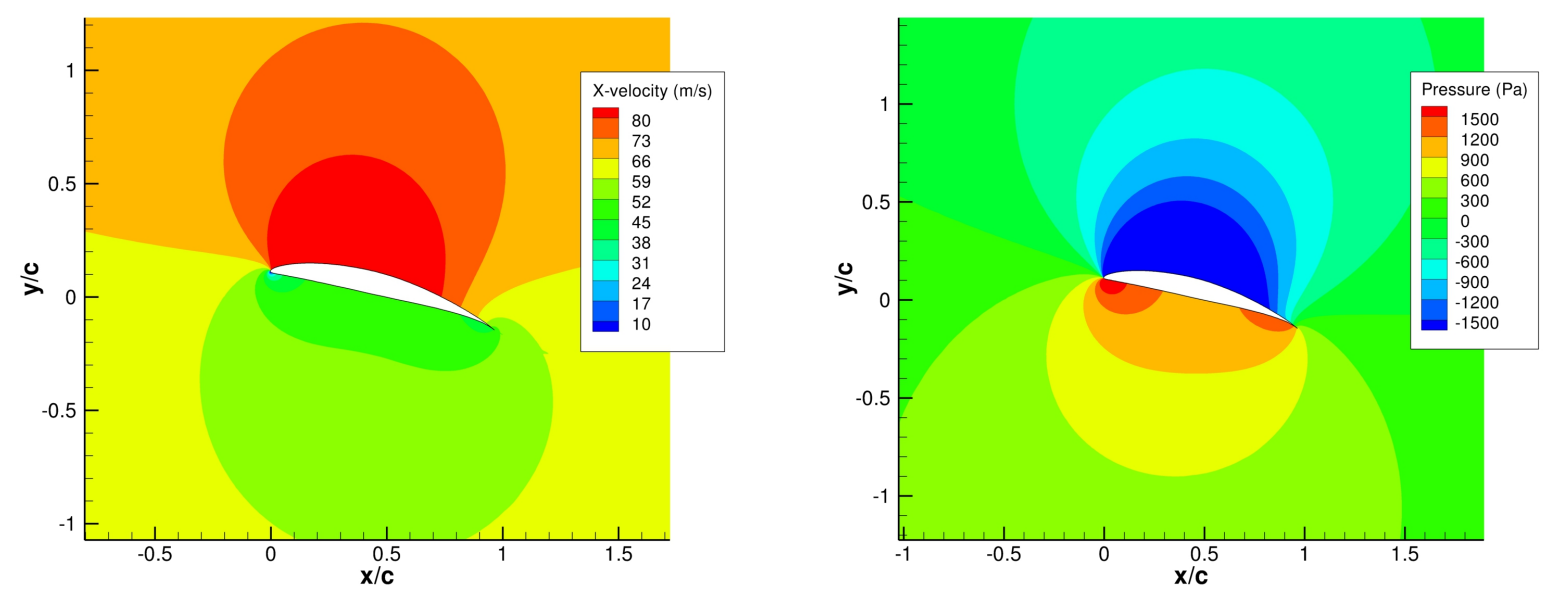

Figure 8: Euler solution on the free stream configuration. (a) Velocity in the $x$-direction and $(b)$ pressure variations around $p_{\infty}=101325 \mathrm{~Pa}$. 
condition also allows hydrodynamic structures to exit the domain. The grid stretching ratio has been kept under $3 \%$ to avoid the appearance of numerical spurious oscillations due to the use of a high-order finite difference scheme. The mesh contains approximately 700000 points and has been split to run parallel computations on 48 processors.

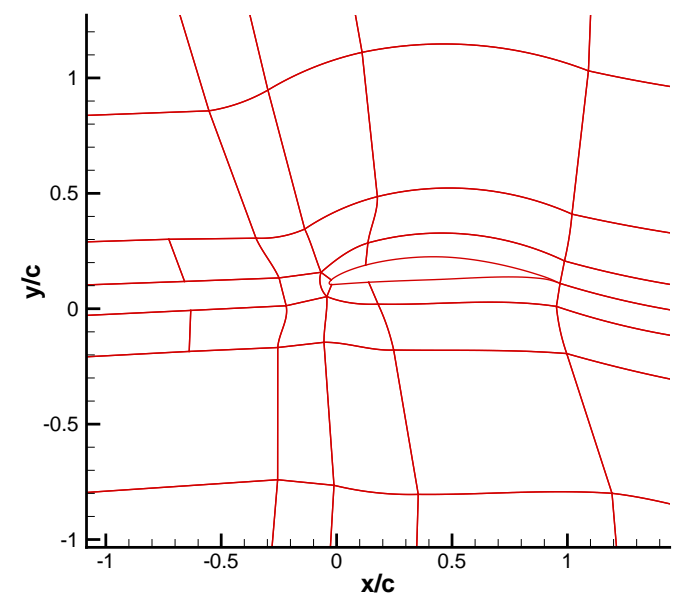

(a) Representation of the mesh splitting into multiple blocks around the airfoil.

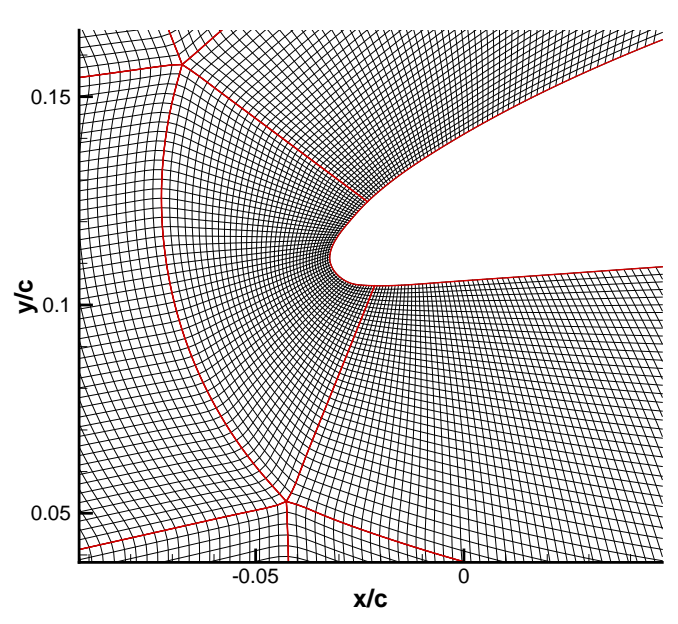

(b) Zoom of the mesh near the leading edge.

Figure 9: CAA mesh. The red lines (-) represent the location of the mesh blocks.

\section{III.B.2. Setup}

The computation is non-dimensionalized using the chord of the airfoil $c=0.15 \mathrm{~m}$, the static speed of sound $a_{\infty}=340 \mathrm{~m} / \mathrm{s}$ and the static density $\rho_{\infty}=1.2 \mathrm{~kg} / \mathrm{m}^{3}$. The non-dimensionalized time step of the simulation is $4.5 .10^{-4}$, corresponding to a maximum local CFL number of 0.9 at the leading edge.

The turbulence is injected through vorticity sources located upstream of the airfoil, at a distance $x_{i}=$ $-2.75 c$ from the leading edge. As discussed in section II.B, a row of vorticity sources is set to generate a turbulence with a constant amplitude over a specific distance in the direction normal to the streamlines. In the present simulation, 50 sources are evenly spaced between $y=-0.25 c$ and $y=0.25 c$. Each source has a half-value radius $b=0.027 c$ and is spaced by a distance $0.01 c$.

The incoming turbulence is modeled by a one-wavenumber von Karman spectrum, using the integral scale and the turbulence intensity extrapolated from the experiments conducted by Gruber et al., ${ }^{22,21}$ respectively $\Lambda=8 \mathrm{~mm}$ and $T_{I}=\sqrt{{\overline{v^{\prime}}}^{2} / u_{\infty}^{2}}=1.7 \%$. It can be expressed by:

$$
\Phi_{w w}\left(k_{x}\right)=\frac{\overline{v^{\prime 2}} \Lambda}{6 \pi} \frac{3+8 \tilde{k}_{x}^{2}}{\left[1+\tilde{k}_{x}^{2}\right]^{11 / 6}} \quad ; \quad \tilde{k}_{x}=\frac{k_{x}}{k_{e}} \quad ; \quad k_{e}=\frac{\sqrt{\pi}}{\Lambda} \frac{\Gamma(5 / 6)}{\Gamma(1 / 3)},
$$

where $\Gamma($.$) is the gamma function. The discretization of the spectrum is realized with a Strouhal number$ step $\Delta \mathrm{St}=0.03125$ for Strouhal numbers between $0.75 \leq S t \leq 25$.

One should note that hydrodynamic modes appear along the airfoil when the gusts are convected through the shear layer in the vicinity of the airfoil. These modes can lead to a divergence of the computation and, if not, they are scattered by the leading edge and generate strong spurious acoustic waves. The method developed here focuses on the leading-edge noise mechanism, and is not able to resolve the turbulent boundary layer on the airfoil. This trailing edge radiation can not be considered as proper trailing-edge noise, and we aim at removing it from the computation before it pollutes the leading-edge radiation. To address this issue, a local sponge zone is applied in the trailing edge region, so that these hydrodynamic modes are dissipated. The value and shape of this local sponge zone have been determined on a flat plate configuration so that its value can damp the instability without affecting the acoustic response. 


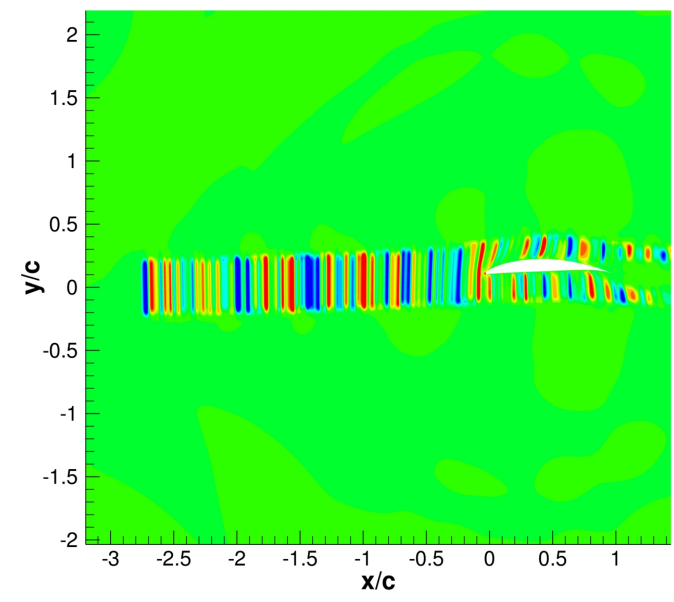

(a) Instantaneous transverse velocity perturbation contour between $\pm 1 m \cdot s^{-1}$.

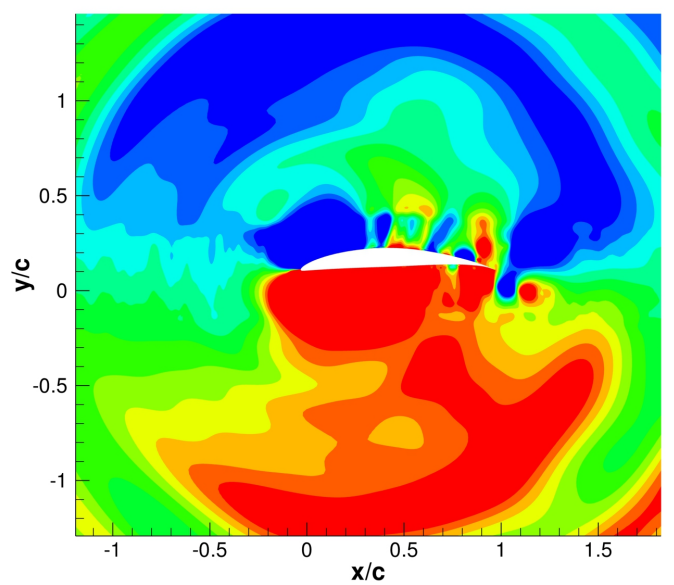

(b) Instantaneous pressure perturbation contour between $\pm 4 \mathrm{~Pa}$.

Figure 10: Instantaneous transverse velocity and pressure perturbation fields.

\section{III.B.3. Far-field radiation}

To estimate the response of the airfoil in the far-field while limiting the size of the computational domain, a Ffowcs-Williams \& Hawkings analogy in the frequency domain ${ }^{24,25}$ is used. This is an exact rearrangement of the general Navier-Stokes equations with the assumption that the source region is limited within a control surface. It simplifies to a convected wave equation with equivalent sources located on the control surface that will generate the same acoustic field as if the full Navier-Stokes equations were solved. These equivalent sources are separated into monopole $Q$, dipole $F$ and quadripole $T$ terms. If the control surface contains all the acoustic sources, the quadripole term can be neglected. Assuming that the control surface is in a uniform translated motion, the integral form of the analogy in the spectral domain can be written: ${ }^{24,25}$

$$
\hat{p}(\mathbf{y}, \omega)=\iint_{S} i \omega \hat{Q}_{n}(\mathbf{x}, \omega) \hat{G}(\mathbf{y}, \omega \mid \mathbf{x}) \mathrm{d} S+\iint_{S} \hat{F}_{i}(\mathbf{x}, \omega) \frac{\partial \hat{G}(\mathbf{y} \mid \mathbf{x}, \omega)}{\partial x_{i}} \mathrm{~d} S
$$

with $\mathbf{y}$ the location of the observer and $\mathbf{x}$ the points on the control surface $S . \hat{G}(\mathbf{y} \mid \mathbf{x}, \omega)$ is the $2 \mathrm{D}$ free-field Green function in the spectral domain for a uniform mean flow in the x-direction, defined as:

$$
\hat{G}(\mathbf{y} \mid \mathbf{x}, \omega)=\frac{i}{4 \beta} H_{0}^{(2)}\left(\frac{k S_{0}}{\beta^{2}}\right) \mathrm{e}^{\frac{i k M}{\beta^{2}}\left(y_{1}-x_{1}\right)}
$$

with $S_{0}=\sqrt{\left(y_{1}-x_{1}\right)^{2}+\beta^{2}\left(y_{2}-x_{2}\right)^{2}}, \beta^{2}=1-M^{2}$ and $H_{0}^{(2)}($.$) the Hankel function of the second kind and$ $0^{\text {th }}$ order. The control surface $S$ has outwards normals $\mathbf{n}$.

- $Q_{n}=\left[\left(\rho_{0}+\rho^{\prime}\right)\left(u_{0 i}+u_{i}^{\prime}\right)-\rho_{0} u_{0 i}\right] n_{i}$ represents the monopolar contribution,

- $F_{i}=\left[\left(p_{0}+p^{\prime}\right) \delta_{i j}+\left(\rho_{0}+\rho^{\prime}\right)\left(u_{i}^{\prime}-u_{0 i}\right)\left(u_{0 j}+u_{j}^{\prime}\right)+\rho_{0} u_{0 i} u_{0 j}\right] n_{j}$ represents the dipolar contribution.

The source terms are calculated in the time domain, and then a Fourier transform is applied to the groupings $Q_{n}$ and $F_{i}$. Moreover, the steady components do not propagate to the far-field and the acoustic propagation is assumed to be linear, hence it can be reduced to $Q_{n}=\left[\rho_{0} u_{i}^{\prime}+\rho^{\prime} u_{0 i}\right] n_{i}$ and $F_{i}=$ $\left[p^{\prime} \delta_{i j}+\left(\rho_{0} u_{i}^{\prime}-\rho^{\prime} u_{0 i}\right) u_{0 j}-\rho_{0} u_{0 i} u_{j}^{\prime}\right] n_{j}$.

\section{III.B.4. Results}

The simulations have been realized considering three mean flow assumptions, namely uniform, inviscid and viscous. After the transitional period, the computations are run over $2^{18}$ iterations, taking about 5 hours 


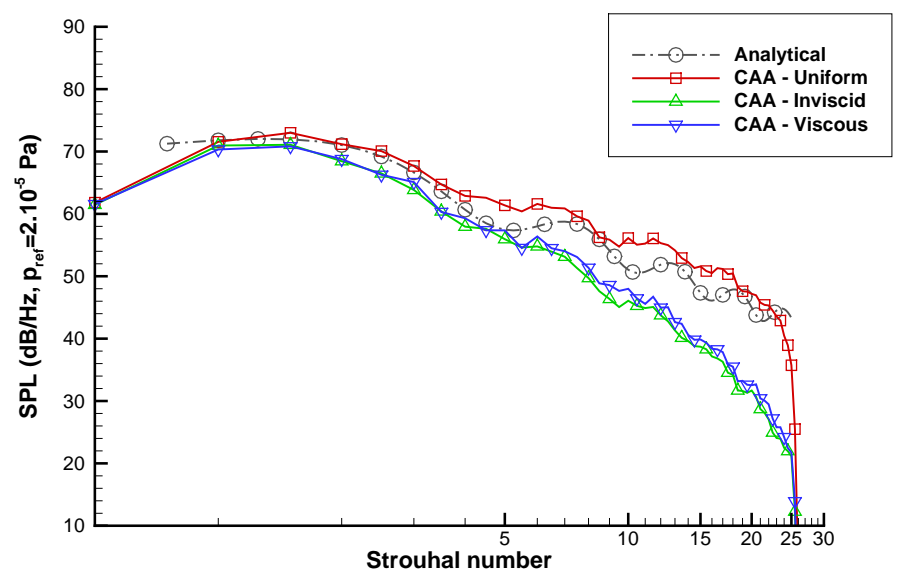

Figure 11: $\mathrm{SPL}$ at $90^{\circ}$ at $\mathrm{R}=1.2 \mathrm{~m}$

on 48 Intel ${ }^{\circledR}$ Xeon ${ }^{\circledR}$ E5-2670 processor cores. The instantaneous normal-velocity and pressure perturbation fields for a viscous mean flow are presented in Figure 10. We can observe the turbulent gusts generated upstream of the profile, in between the inlet boundary and the leading edge, with a limited extent in the y-direction, and being convected by the mean flow. The pressure fluctuations show an expected dipolar pattern of the acoustic response of the airfoil. As mentioned before, hydrodynamic modes are generated along the airfoil for this simulation using the viscous mean flow, but the local sponge layer seems to prevent them from radiating when they convect through the trailing edge.

A set of 720 equally spaced sensors are located on a circle centered on the leading edge, with a radius of 1.7 chords, to record the perturbations and perform the FWH analogy (described in section III.B.3). The numerical predictions are compared with the analytical results of the Amiet model which considers a flat plate. ${ }^{11}$ Figure 11 shows the Sound Pressure Level (SPL) at $1.2 \mathrm{~m}$ and $90^{\circ}$ (above the airfoil), for the three mean flow configurations, and the Amiet model. The spectra are averaged on 15 segments using a Hann window and an overlapping of $50 \%$. We can see that the mean flow have a very little effect on low frequencies, but as the frequency increases, the rate of decay is more pronounced for the inviscid and viscous mean flows. Indeed, the shear layers in the vicinity of the airfoil (especially close to the stagnation point) in these two configurations mainly affect the small wavelengths. One can note that the results obtained with the viscous and inviscid mean flows are slightly underestimated at low frequency, which requires further investigation. The difference between the inviscid and viscous mean flows is negligible at low frequencies and remain very small at higher frequencies. This is due to the fact that the differences between inviscid and viscous flows are restricted to the boundary layer region, which has a relatively weak influence on the noise generation mechanism.

At high frequencies, the upstream directivities (Figure 12) computed from the simulations, are tilted compared with the Amiet solution. The angle between the local mean flow velocity and the camber line of the airfoil can explain this tilt. In section IV.A, some investigation is done regarding the change in angle of attack.

\section{III.B.5. Comparaison with experiments}

The numerical results obtained are compared to the ISVR-rig measurements performed by Paruchuri. To compare the numerical results obtained from a 2D simulation with experiments, two elements have to be taken into consideration. Firstly, the acoustic propagation differs in 3D, as the acoustic energy also propagates in the third dimension. Fortunately, if the 3D effects are low (it is the case for an airfoil with a span large relative to the chord), the pressure fluctuations obtained from a 2D simulation can be duplicated along the span to have a 3D configuration, resulting in fully correlated sources in the span direction. Dieste et al. ${ }^{9}$ then deduced a correction to estimate a 3D Spp from a 2D result by comparing the 2D and 3D Amiet 


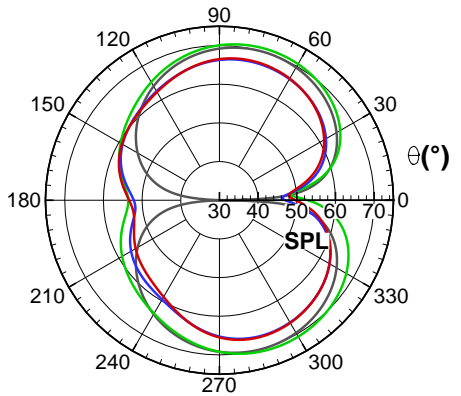

(a) $\mathrm{St}=2.5$

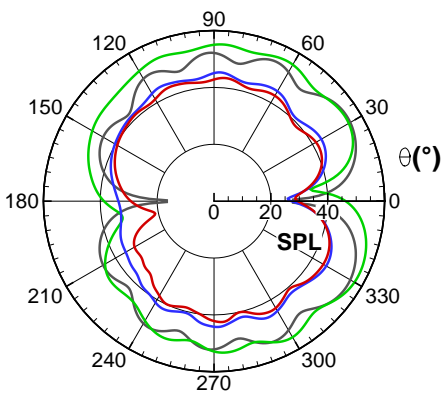

(b) $\mathrm{St}=12.5$

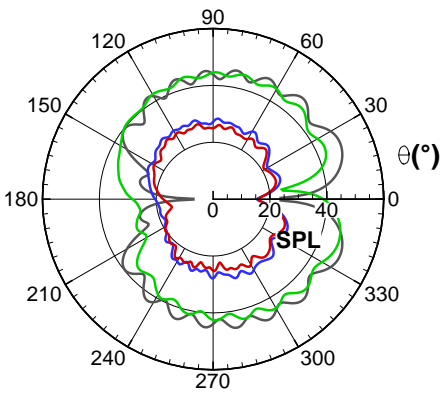

(c) $\mathrm{St}=22.5$

Figure 12: Directivity at $\mathrm{R}=1.2 \mathrm{~m}$ using a uniform ( $(-)$, inviscid ( - ) and viscous ( - ) mean flow assumption, against the Amiet model for a flat plate (-). SPL plotted in $\mathrm{dB} / \mathrm{Hz}$, using a reference pressure $p_{\text {ref }}=2 \cdot 10^{-5} \mathrm{~Pa}$.

formulations:

$$
S_{p p}^{3 D}(x, y, 0, \omega)=\frac{k l_{y}(\omega) L}{\pi \sigma} S_{p p}^{2 D}(x, y, \omega) \quad \text { with } l_{y}(\omega)=\frac{8 \Lambda}{3}\left(\frac{\Gamma(1 / 3)}{\Gamma(5 / 6)}\right)^{2} \frac{\tilde{k}_{x}^{2}}{\left(3+8 \tilde{k}_{x}^{2}\right) \sqrt{1+\tilde{k}_{x}^{2}}}
$$

where $\omega$ is the angular frequency, $k=\omega / c_{0}$ is the free-field acoustic wavenumber, $k_{x}=\omega / u_{0}$ is the hydrodynamic wavenumber in the streamwise direction, $\mathrm{L}$ is the span of the airfoil, $\sigma=\sqrt{x^{2}+\left(1-M^{2}\right) y^{2}} \cdot l_{y}(\omega)$ is the span-wise correlation length scale, which is known for the von Karman model used in our simulations. The second effect to account for is due to the setup of the experiments. The jet flow of the test-rig has a finite height, so there is a shear layer between the airfoil and the microphones. This shear layer has a refraction effect, which affects the radiation direction and the amplitude of the acoustic waves. In the present work, the corrections proposed by Amiet ${ }^{26}$ have been used.

The experimental measurements can only be compared to the simulation within a limited extent of the spectrum where leading-edge noise is dominant. At low frequency, noise generated by the turbulent grid as well as the jet noise are dominant, while at high frequency, the self-noise (or trailing-edge noise) becomes dominant.

The SPL computed from the numerical solutions are compared to the experimental results in Figure 13. The low and high frequency limits mentioned before, where the leading edge noise is dominant are represented by the vertical dashed lines. The $3 \mathrm{~dB}$ underestimation observed in previous section at low frequency when compared with the Amiet solution of a flat plate is also found in the comparison with the experimental data, but on the whole spectrum at $90^{\circ}$. Yet, upstream, the numerical predictions show a good agreement with experiments. The experimental data can not be compared further downstream as the microphones are located in the jet stream. Similar observations can be made on the directivities plotted in Figure 14 at different frequencies. 


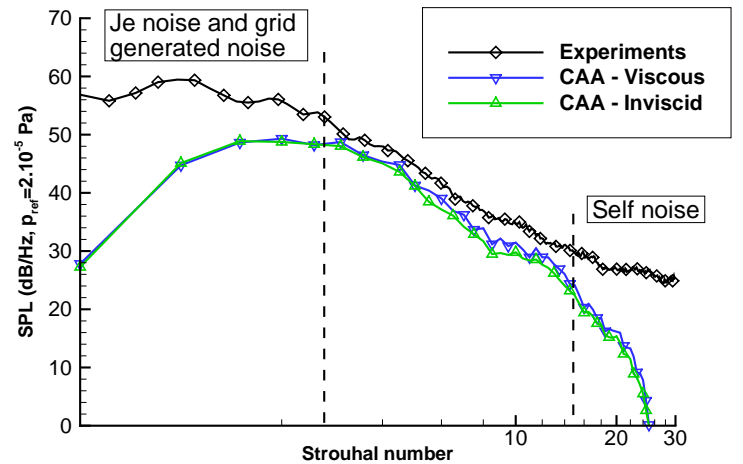

(a) $70^{\circ}$

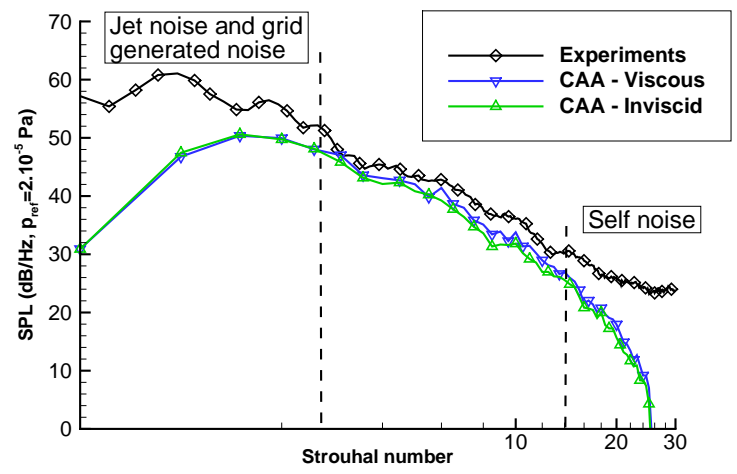

(c) $110^{\circ}$

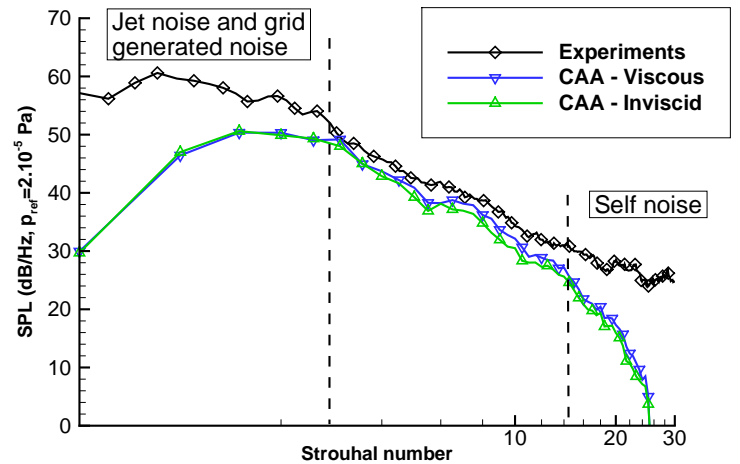

(b) $90^{\circ}$

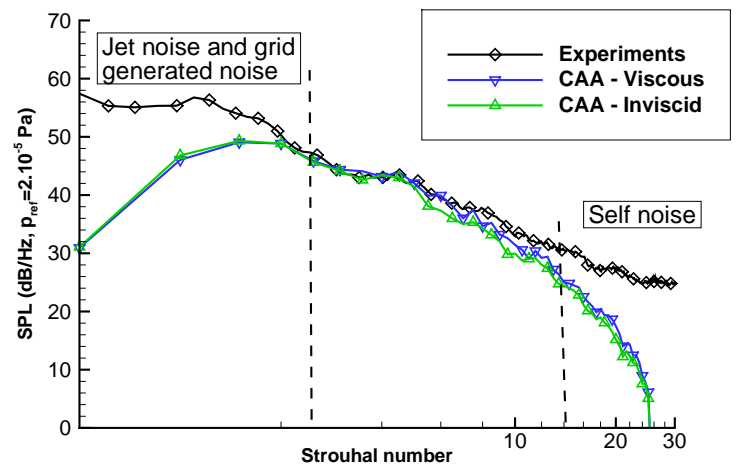

(d) $130^{\circ}$

Figure 13: Comparison of the numerical predictions using the inviscid and viscous mean flow configuration with experimental measurements.

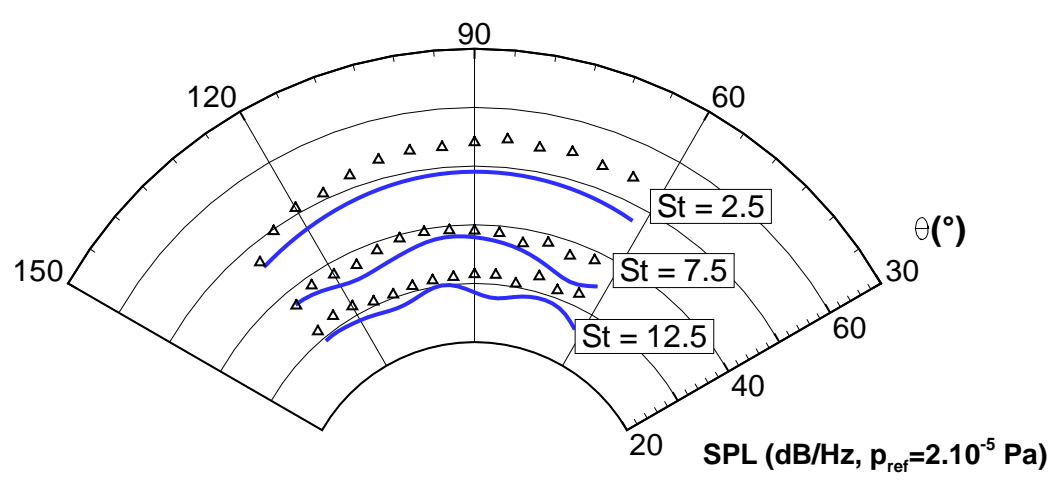

Figure 14: Directivity of the numerical predictions using the inviscid mean flow configuration $(\longrightarrow)$ with experimental measurements $(\Delta)$.

\section{Parametric study}

In this section, the effects of the angle of attack (AoA), the integral length scale $\Lambda$ (IS) and the mean flow velocity $u_{0}$ are studied. 


\section{IV.A. Variation of the angle of attack}

Simulations have been run for several angles of attack between $0^{\circ}$ and $3^{\circ}$, using the same airfoil and flow conditions than before. For higher AoA, some numerical instabilities appeared on the upper surface of the airfoil with both the viscous and inviscid mean flows.

The SPL at $90^{\circ}$ and $R=1.2 \mathrm{~m}$ are plotted in Figure 15 for the viscous flow calculations. They reveal a reduction of the noise radiated at high frequencies when the AoA is increased, as it has been observed experimentally. ${ }^{21}$ The reduction visible here is nonetheless higher than the one observed experimentally. It corroborates the assumption made by Gill ${ }^{12}$ that a one-component turbulence is not satisfactory to correctly predict the radiated acoustics of a profile with an AoA.

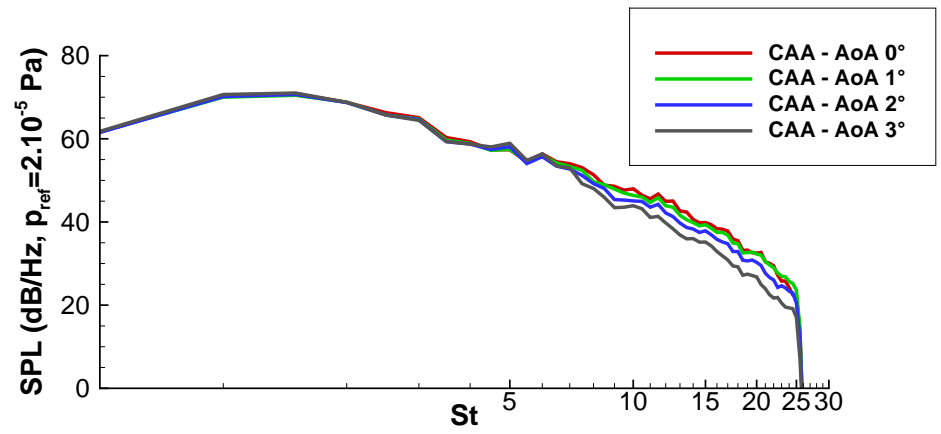

Figure 15: Comparison of the SPL at $90^{\circ}$ at $\mathrm{R}=1.2 \mathrm{~m}$ for an AoA of $0^{\circ}(\square), 1^{\circ}(\square), 2^{\circ}(\square)$ and $3^{\circ}(-)$.

On Figure 16, the directivities show very little differences at low frequency at all angles, yet at high frequency, the upstream minimum is tilted in the clockwise direction as the AoA increases and it reaches $\theta=0^{\circ}$ for the airfoil with $3^{\circ}$ AoA for the highest frequency (Figure 16c). It is probably due to the fact that the steepest AoA considered is close to a zero angle formed between the local mean flow velocity and the camber line of the airfoil, as previously mentioned in section III.

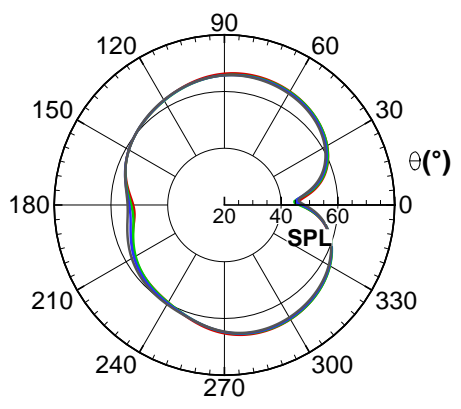

(a) $\mathrm{St}=2.5$

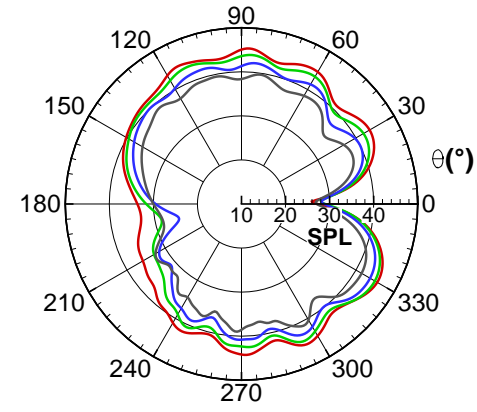

(b) $\mathrm{St}=12.5$

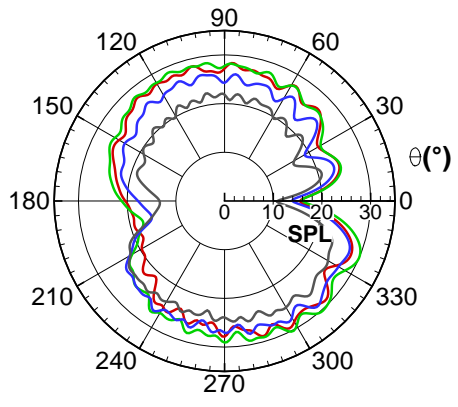

(c) $\mathrm{St}=22.5$

Figure 16: Directivity at $\mathrm{R}=1.2 \mathrm{~m}$ for an AoA of $0^{\circ}(-), 1^{\circ}(-), 2^{\circ}(-)$ and $3^{\circ}(-)$. SPL plotted in $\mathrm{dB} / \mathrm{Hz}$, using a reference pressure $p_{\text {ref }}=2 \cdot 10^{-5} \mathrm{~Pa}$.

\section{IV.B. Variation of the integral length scale}

In the present paper, the turbulence is considered isentropic and follows a von Karman energy spectrum, hence it can be fully characterized by a turbulent intensity and an integral length scale. The evolution of the spectrum with the turbulent intensity is linear, therefore easy to predict, whereas a change of the integral length scale leads to change the rate of the energy decay. In this paragraph, the impact of the integral length scale on the noise radiated is studied using three different values: $0.004 \mathrm{~m}, 0.008 \mathrm{~m}$ and $0.012 \mathrm{~m}$. The other parameters remain the same as for the validation case with a viscous mean flow (section III).

Following Amiet's model ${ }^{11}$ for a flat plate, the chordwise integral of the surface loading of a flat plate 
with a chord $c$ and a span $2 d$ is defined as:

$$
\mathcal{L}\left(x, K_{x}, k_{y}\right)=\int_{-c / 2}^{c / 2} g\left(x, K_{x}, k_{y}\right) \mathrm{e}^{-i \omega x_{0}(M-x / \sigma) / a_{\infty} \beta^{2}} \mathrm{~d} x_{0},
$$

where $\beta=\sqrt{1-M^{2}}, \sigma=\sqrt{x^{2}+\beta^{2}\left(y^{2}+z^{2}\right)}$ and $g\left(x, K_{x}, k_{y}\right)$ is the transfer function between turbulent velocity and flat plate pressure jump. The far-field PSD of a flat plate interacting with turbulent gusts can be written as: ${ }^{11}$

$$
S_{p p}(x, y, z, \omega)=\left(\frac{\omega z \rho_{\infty} c}{2 a_{\infty} \sigma^{2}}\right)^{2} U d \pi \int_{-\infty}^{\infty}\left[\frac{\sin ^{2}\left(d\left(k_{y}+\omega y / a_{\infty} \sigma\right)\right)}{\left(k_{y}+\omega y / a_{\infty} \sigma\right)^{2} \pi d}\right]\left|\mathcal{L}\left(x, K_{x}, k_{y}\right)\right|^{2} \Phi_{w w}\left(K_{x}, k_{y}\right) \mathrm{d} k_{y} .
$$

We can observe in equation (12) that the loading function of the flat plate is independent from the incoming turbulence spectrum. Hence, for a flat plate, the evolution of the radiated noise spectra when the integral length scale changes are only related to the changes of the turbulence spectrum, and the loading function remains identical. The comparison (Figure 17) of the $\Delta \mathrm{SPL}$ for different IS $\left(\Delta \mathrm{SPL}_{\mathrm{IS}}=\mathrm{SPL}_{\mathrm{IS}_{\mathrm{ref}}}-\right.$ $\mathrm{SPL}_{\mathrm{IS}}$ with $\mathrm{IS}_{\text {ref }}=8 \mathrm{~mm}$ ) reveals similar trends between the numerical predictions and the Amiet model. Moreover, as showed in Figure 18, the pattern of the directivity remain unchanged, showing that, as for a flat plate, the loading of an airfoil is independent from the incoming turbulence and thus, that the changes in the acoustic radiation are only related to the changes in the spectrum of the incoming turbulence.

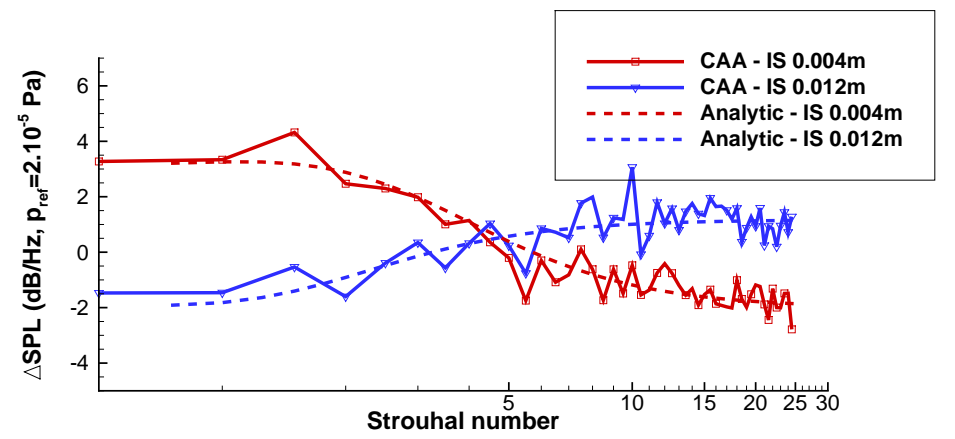

Figure 17: Comparison of the $\Delta$ SPL for different IS. $\Delta \mathrm{SPL}_{\mathrm{IS}}=\mathrm{SPL}_{\mathrm{IS}_{\mathrm{ref}}}-\mathrm{SPL}_{\mathrm{IS}}$ with $\mathrm{IS}_{\mathrm{ref}}=8 \mathrm{~mm}$. The dashed lines are the results for the Amiet model and the continuous lines are for the CAA predictions.

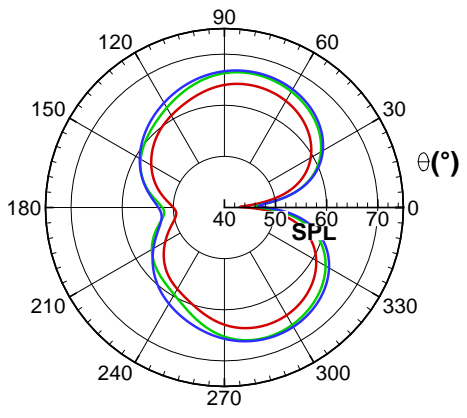

(a) $\mathrm{St}=2.5$

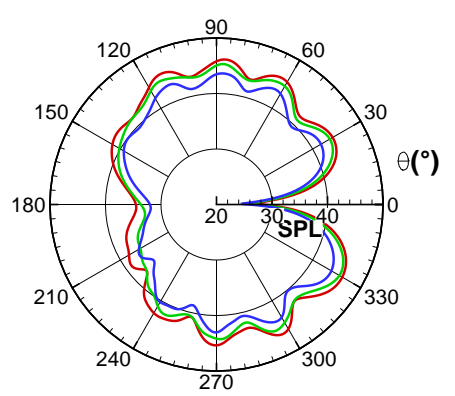

(b) $\mathrm{St}=12.5$

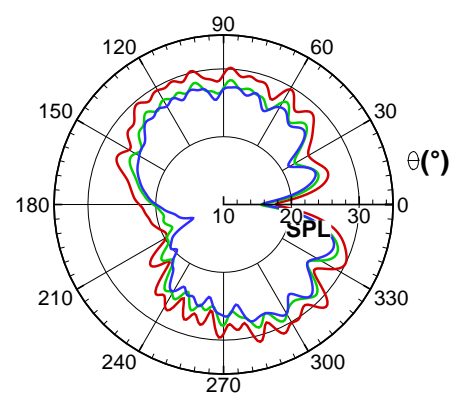

(c) $\mathrm{St}=22.5$

Figure 18: Directivity at $\mathrm{R}=1.2 \mathrm{~m}$ with IS $=0.004 \mathrm{~m}(\square)$, IS $=0.008 \mathrm{~m}(-)$ and IS $=0.012 \mathrm{~m}(-)$. SPL plotted in $\mathrm{dB} / \mathrm{Hz}$, using a reference pressure $p_{\text {ref }}=2.10^{-5} \mathrm{~Pa}$.

\section{IV.C. Variation of the mean flow velocity}

In this section, the effect of the mean flow velocity is investigated. To prevent instabilities due to the presence of boundary layers, the numerical predictions have been realized using a uniform and an inviscid mean flow assumption. Moreover, to correctly support the hydrodynamic and acoustic structures, the injected turbulence is limited $7500 \mathrm{~Hz}$. 


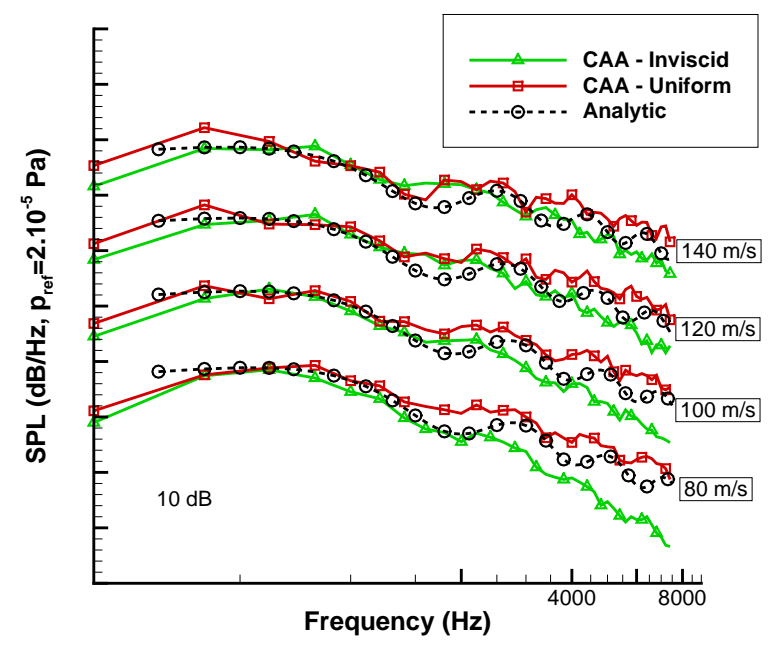

Figure 19: Comparison of the OASPL of the numerical predictions with multiple mean flow velocities, with the Amiet model of a flat plate, at $90^{\circ}$ and $\mathrm{R}=1.2 \mathrm{~m}$

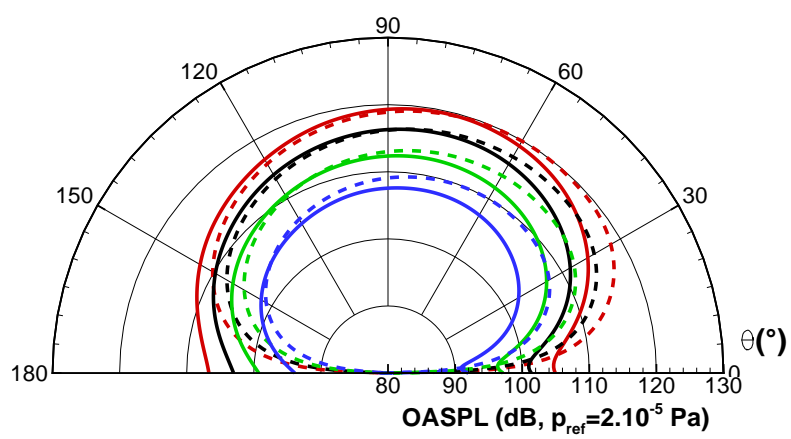

Figure 20: Comparison of the OASPL of the numerical predictions (in continuous line), with the Amiet model of a flat plate (in dashed line), for a mean flow velocity of $80 \mathrm{~m} / \mathrm{s}(-), 100 \mathrm{~m} / \mathrm{s}(-), 120 \mathrm{~m} / \mathrm{s}$ (一) and $140 \mathrm{~m} / \mathrm{s}(\longrightarrow)$. 
The Figure 19 compares the numerical predictions with multiple mean flow velocities, with the Amiet solutions for a flat plate, at $90^{\circ}$, at a distance of $1.2 \mathrm{~m}$. It shows that the difference of the acoustic spectra observed at high frequencies between the predictions for a NACA 65(12)10 airfoil and the analytical solution of a flat plate, is less important as the mean flow velocity increases. The Overall Sound Pressure Level (OASPL), plotted in Figure 20, confirms this observation. In the litterature, similar trends have been observed on symetric profiles by Paterson \& Amiet $^{27}$ on a NACA 0012 airfoil or Kim et al. ${ }^{28}$ on NACA 0012 and NACA 0015 airfoils.

\section{Conclusion}

A new method to inject synthetic turbulence in a computational domain using localized vorticity sources has been developed. This method has the advantages of being easy to implement and has no influence on the parallelization of the solver, whilst the turbulence generated is frozen. The method has been firstly validated in a free-field configuration considering a uniform mean flow and using a CAA method solving the linearized Euler equations. Then, the method is applied to predict the noise resulting from the interaction of an incoming turbulence and a 2D isolated NACA 65(12)10 airfoil with uniform, inviscid and viscous mean flows. These computations are part of the Fundamental test Case 1 (FC1) of the Fan Stage Broadband Noise Benchmarking Programme. The acoustic spectra are compared with ISVR-rig measurements performed by Paruchuri and show a good agreement. The results indicate that the differences in the leading edge predictions using inviscid and viscous mean flows are negligible at low frequency and remain very limited at higher frequencies. This is due to the fact that the differences between inviscid and viscous flows are restricted to the boundary layer region, which has a relatively weak influence on the noise generation mechanism. The study of the effect of the angle of attack suggests that a one-component turbulent model is not satisfactory to perform accurate acoustic predictions with an angle of attack as it overestimates the rate of decay at high frequencies. The changes in the radiated noise with the integral length for an airfoil display trends that are similar to the one observed for a flat plate, confirming that the loading of the airfoil is independent from the incoming turbulence and thus, that the changes in the radiated noise are directly related to the changes in the turbulence spectrum. Finally, it has been found that the higher the mean flow velocity is, the closer the numerical predictions are to the Amiet model for a flat plate. The next step of this study will be the realization of complementary simulations to provide a better insight on the physics of interaction noise. This involves the extension of the developed method to consider two-component turbulence and also to consider $3 \mathrm{D}$ airfoil geometry configurations.

\section{Acknowledgements}

The work was funded by Innovate UK, HARMONY Programme (GA n 101367). Rolls-Royce Plc is also acknowledged for the financial and technical support given.

The authors would like to thank the DLR for providing the CAA solver PIANO. The authors would also like to thank Chaitanya Paruchuri and Dr. Phillip Joseph for providing ISVR-rig measurements.

\section{References}

${ }^{1}$ P. R. Gliebe and B. A. Janardan. Ultra-high bypass engine aeroacoustic study. Technical report, NASA, 2003.

${ }^{2}$ R. Kraichnan. Diffusion by a random velocity field. Physics of Fluids, 13(1):22-31, 1970.

${ }^{3}$ R. Ewert and W. Schröder. Acoustic perturbation equations based on flow decomposition via source filtering. J. Comput. Phys., 188:365-398, 2003.

${ }^{4} \mathrm{M}$. Dieste and G. Gabard. Broadband fan interaction noise using synthetic inhomogeneous non-stationary turbulence. In 17th AIAA/CEAS Aeroacoustics Conference, number AIAA-2009-3267, 2011.

${ }^{5}$ M. Dieste and G. Gabard. Random particle methods applied to broadband fan interaction noise. Journal of Computational Physics, 231:8133-8151, 2012.

${ }^{6}$ N. Jarrin, S. Benhamadouche, D. Laurence, and R. Prosser. A synthetic-eddy method for generating inflow conditions for large-eddy simulations. International Journal of Heat and Fluid Flow, 27:585-593, 2006.

${ }^{7}$ N. Jarrin, J-C. Uribe, R. Prosser, and D. Laurence. Advances in Hybrid RANS-LES Modelling, volume 97, chapter Synthetic Inflow Boundary Conditions for Wall Bounded Flows, pages 77-86. Springer, 2008.

${ }^{8}$ N. Jarrin, R. Prosser, J-C. Uribe, S. Benhamadouche, and D. Laurence. Reconstruction of turbulent fluctuations for hybrid rans/les simulations using a synthetic-eddy method. International Journal of Heat and Fluid Flow, 30:435-442, 2009.

${ }^{9}$ M. Dieste. Random-Vortex-Particle Methods Applied to Broadband Fan Interaction Noise. PhD thesis, University of 
Southampton, 2011.

${ }^{10}$ V. Clair. Calcul numrique de la rponse acoustique d'un aubage soumis un sillage turbulent. PhD thesis, Universit Claude Bernard Lyon 1, 2013.

${ }^{11}$ R.K. Amiet. Acoustic radiation from an airfoil in turbulent stream. Journal of Sound and Vibration, 41(4):407-420, 1975.

12 J. Gill. Broadband Noise Generation of a Contra-Rotating Open Rotor Blade. PhD thesis, Airbus Noise Technology Centre, University of Southampton, 2015.

${ }^{13} \mathrm{~J}$. Coupland. Fan stage broadband noise benchmarking programme - specification of fundamental test case 1 (fc1). http://www.oai.org/aeroacoustics/FBNWorkshop, 2014.

${ }^{14}$ B.-T. Chu and L. Kovásznay. Non-linear interactions in a viscous heat-conducting compressible gas. Journal of Fluid Mechanics, 3(5):494-514, 1958.

${ }^{15} \mathrm{C}$. Tam and J. Webb. Dispersion-Relation-Preserving finite difference schemes for computational acoustics. Journal of Computational Physics, 107:262-281, 1993.

${ }^{16}$ J.M. Nichols, C.C. Olson, J.V. Michalowicz, and F. Bucholtz. A simple algorithm for generating spectrally colored, non-gaussian signals. Probabilistic Engineering Mechanics, 25:315-322, 2010.

${ }^{17}$ F. Elliott, D. Horntrop, and A. Majda. A fourier-wavelet monte carlo method for fractal random fields. Journal of Computational Physics, 132:384-408, 1997.

${ }^{18}$ G. Gabard. Noise sources for duct acoustics simulations: Broadband noise and tones. AIAA Journal, 52:1994-2006, 2014.

${ }^{19}$ Stephane Moreau, Manuel Henner, Gianluca Iaccarino, Meng Wang, and Michel Roger. Analysis of flow conditions in freejet experiments for studying airfoil self-noise. AIA A Journal, 41(10):1895-1905, 2003.

${ }^{20}$ H. Deniau, J.-F. Boussuge, C. Polacsek, and S. Moreau. Affordable compressible LES of airfoil-turbulence interaction in a free jet. In 17th AIAA/CEAS Aeroacoustics Conference, number AIAA-2011-2707, 2011.

${ }^{21}$ M. Gruber. Airfoil noise reduction by edge treatments. PhD thesis, University of Southampton, ISVR, 2012.

${ }^{22} \mathrm{M}$. Gruber, P. Joseph, and T.P. Chong. On the mechanisms of serrated airfoil trailing edge noise reduction. In $17 t h$ AIAA/CEAS Aeroacoustics Conference, number AIAA 2011-2781, 2011.

${ }^{23}$ V. Clair, C. Polacsek, T. Le Garrec, and G. Reboul. CAA methodology to simulate turbulence-airfoil noise. In Proceedings of the 18th AIAA/CEAS Aeroacoustics Conference, number AIAA-2012-2189, 2012.

${ }^{24}$ David P. Lockard. An efficient, two-dimensional implementation of the Ffowcs-Williams and Hawkings equation. Journal of Sound and Vibration, 229(4):897-911, 2000.

${ }^{25}$ David P. Lockard. A comparison of Ffowcs Williams-Hawkings solvers for airframe noise applications. In 8 th AIAA/CEAS Aeroacoustics Conference, number AIAA-2002-2580, 2002.

${ }^{26} \mathrm{R}$. Amiet. Refraction of sound by a shear layer. Journal of Sound and Vibration, 54(4):467-482, 1978.

${ }^{27}$ R. Paterson and R. Amiet. Acoustic radiation and surface pressure characteristics of an airfoil due to incident turbulence. NASA Contractor Report 2733, 1976.

${ }^{28}$ Daehwan Kim; Gwang-Se Lee; Cheolung Cheong. Inflow broadband noise from an isolated symmetric airfoil interacting with incident turbulence. Journal of Fluids and Structures, 2015. 